\title{
Wakeup Scheduling for Energy-Efficient Communication in Opportunistic Mobile Networks
}

\author{
Wei $\mathrm{Gao}^{*}$ and Qinghua $\mathrm{Li}^{\dagger}$ \\ ${ }^{*}$ Department of Electrical Engineering and Computer Science, University of Tennessee, Knoxville \\ ${ }^{\dagger}$ Department of Computer Science and Engineering, The Pennsylvania State University \\ *weigao@utk.edu, ${ }^{\dagger}$ qxl118@cse.psu.edu
}

\begin{abstract}
Opportunistic mobile networks consist of mobile devices which only communicate when they opportunistically contact each other. Periodic contact probing is required to facilitate opportunistic communication, but seriously reduces the limited battery life of mobile devices. Current research efforts on reducing energy consumption of contact probing are restricted to optimize the probing interval, but are insufficient for energy-efficient opportunistic communication. In this paper, we propose novel techniques to adaptively schedule wakeup periods of mobile nodes between their inter-contact times. A node stays asleep during inter-contact times when contact probing is unnecessary, and only wakes up when a contact with another node is likely to happen. Our approach probabilistically predicts node contacts in the future, and analytically balances between energy consumption for contact probing and performance of opportunistic communication. Extensive trace-driven simulations show that our approach significantly improves energy efficiency of opportunistic communication compared to existing schemes.
\end{abstract}

\section{INTRODUCTION}

Opportunistic Mobile Networks, which are also known as Delay Tolerant Networks (DTNs) [9] or Pocket Switched Networks (PSNs) [14], consist of hand-held mobile devices such as PDAs, laptops, and smartphones. These devices are connected only intermittently when they opportunistically contact each other, i.e., move into the communication range of their short-range radios (e.g., Bluetooth, WiFi). Such intermittent network connectivity makes it difficult to maintain end-toend communication links among mobile nodes. Instead, node mobility is exploited to let nodes physically carry data as relays, which forward data when they opportunistically contact other nodes. Extensive research efforts have been focusing on how to make effective forwarding decisions upon opportunistic contacts, to ensure that data is carried by relays with the best chance to contact the destinations [21], [1], [24], [12].

To facilitate opportunistic communication, each node periodically probes contacts with its peers nearby. Experimental studies [2], [22] have shown that mobile nodes devote the majority of their consumed energy to periodic contact probing rather than actual data transfer. Therefore, appropriate energy saving schemes are essential for improving the energy efficiency of opportunistic communication, and enabling mobile devices with a limited battery life to avoid unnecessary contact probing while preserving opportunistic communication performance. Existing work suggested that mobile nodes only wake up at scheduled contact probing times, and stay asleep during the meantime [23], [4]. The energy efficiency of opportunistic communication can hence be improved by optimizing the interval of contact probing [22].

In this paper, we observe that the existing work is insufficient for energy-efficient opportunistic communication, because they only avoid repetitively probing a contact within the duration of that contact. Comparatively, mobile nodes waste the majority of their energy on unnecessary contact probing during inter-contact times, which are usually much longer than contact durations in realistic mobile networks.

The major focus of this paper, therefore, is to adaptively schedule wakeup periods of mobile nodes between their intercontact times, to ensure that a node stays asleep during its inter-contact times and only wakes up when a contact with another node is likely to happen. During each wakeup period, a node probes its vicinity with the optimal probing interval suggested by the existing work. Our basic idea is to probabilistically predict node contacts based on stochastic modeling of node contact process. Based on the prediction results, our wakeup scheduling scheme balances between energy consumption of contact probing and opportunistic communication performance. To the best of our knowledge, we are the first to intentionally study energy saving during inter-contact times in opportunistic mobile networks.

Our detailed contributions are as follows:

- We propose a probabilistic framework for wakeup scheduling between a pair of nodes which contact each other. This framework enables analytical optimization of energy consumption for contact probing, based on the requirements of opportunistic communication performance.

- We integrate the pairwise wakeup schedules of a specific node with other nodes to generate its cumulative wakeup schedule, and further optimize the energy efficiency of opportunistic communication during such integration. We also preserve the scheduling consistency during such integration via efficient coordination among mobile nodes, to ensure that two nodes stay awake simultaneously each time when they contact each other.

- We develop techniques to effectively handle the possible inaccuracy of contact prediction in wakeup scheduling, and analytically prevent such inaccuracy from degrading the performance of opportunistic communication.

The rest of this paper is organized is follows. Section II briefly reviews existing work. Section III provides an overview of our approach and highlights our motivation of wakeup scheduling between inter-contact times. Section IV describes wakeup scheduling between a pair of nodes, and 


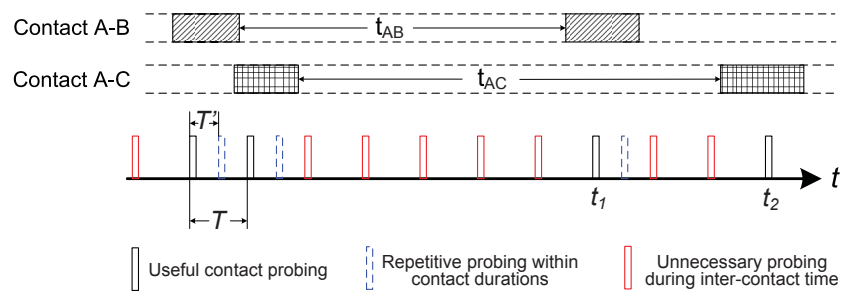

Fig. 1. Energy consumption of periodic contact probing. Existing energy saving schemes only avoid repetitive contact probing within contact durations, but ignore more unnecessary contact probing during inter-contact times.

in Section $\mathrm{V}$ we integrate the pairwise wakeup periods to generate cumulative wakeup schedules. Results of trace-driven performance evaluations are presented in Section VI. Section VII discusses the paper and Section VIII concludes the paper.

\section{RELATED WORK}

Nodes in opportunistic mobile networks communicate with each other in a "carry-and-forward" manner, and forward data when they opportunistically contact others. For such forwarding decision, specific metrics are developed to evaluate a node's capability of contacting others, based on the node's mobility pattern [24], stochastic contact process [1], or social behaviors [15], [12]. These metrics are applied to various routing strategies [5], [21], [8] with different tradeoffs between communication performance and overhead, and are also applied to other communication paradigms, such as data dissemination [17] and cooperative caching [11].

Energy saving in opportunistic mobile networks is motivated by the limited battery life of mobile devices and periodic contact probing required for opportunistic communication. Some studies reduce energy consumption by limiting the number of duplicate data copies created in the network [21], [18]. Later studies reduce energy consumption of periodic contact probing by optimizing the probing interval [22] or employing low-duty-cycle techniques [13]. They suggest that nodes stay asleep most of time and only wake up at scheduled contact probing times [23], [4]. In all these schemes, energy consumption is only reduced within contact durations which are usually much shorter than inter-contact times.

Instead of using fixed wakeup schedules, mobile nodes can also be waken up in an on-demand manner, with a secondary low-power, long-range radio such as XTend [2] or CC1000 [16]. These radios are used to pre-detect contacts before a node comes into the communication range of WiFi or Bluetooth radios, which are then waken up for possible data transfer. However, the applicability of these systems is limited in practice due to the requirement of secondary radio which is usually unavailable on personal mobile devices. Comparatively, in this paper we propose real-time wakeup scheduling techniques based on the results of contact prediction, without any modifications on the existing hardware.

\section{OVERVIEW}

\section{A. Motivation}

Recent technical advance of smartphones, which are capable of processing various media contents, motivate applications of opportunistic mobile networks including mobile data sharing [19], urban sensing [7], and disaster recovery. We experimentally examine the average energy consumption of Samsung Nexus S smartphone with different tasks during 5 minutes. Experimental results listed in Table I show that contact probing via Bluetooth or WiFi radios on smartphones consumes as much energy as that of watching videos or making phone calls. Hence, unnecessary contact probing significantly reduces the battery life of smartphones if not being appropriately avoided.

TABLE I

ENERGY CONSUMPTION OF SMARTPHONES

\begin{tabular}{c|cc}
\hline Task & Mean power $(\mathrm{mW})$ & Variance \\
\hline \hline Idle & 23.4664 & 3.0688 \\
Text reading & 66.4898 & 10.2445 \\
Video playback & 209.4283 & 88.5421 \\
3G Phone call & 169.2245 & 52.5547 \\
\hline Bluetooth on & 25.2297 & 1.8442 \\
Bluetooth probing & 157.5559 & 71.2458 \\
WiFi probing & 221.4587 & 75.2487 \\
\hline
\end{tabular}

Current schemes [23], [22], [4] reduce energy consumption of contact probing by optimizing the probing interval $(T)$, so as to avoid repetitively probing a contact within the duration of that contact. As shown in Figure 1, the length of the optimized interval $T$ can be as twice as that of the original interval $T^{\prime}$.

However, existing schemes are insufficient for energyefficient communication in opportunistic mobile networks, where inter-contact time is usually much longer than contact durations. This is demonstrated by real-world network traces summarized in Table II, which record contacts among mobile devices at conference site (Infocom [15]) or university campuses (MIT Reality [6], UCSD [20]). As illustrated in Figure 1 , before node $A$ detects its next contacts with $B$ and $C$ at time $t_{1}$ and $t_{2}$ respectively, many unnecessary contact probing happens during inter-contact times $t_{A B}$ and $t_{A C}$. Obviously, such amount of consumed energy is much larger than that within contact durations, and can be as much as 100 times in the MIT Reality trace as shown by Table II.

Our basic solution to address this insufficiency is to probabilistically predict node contacts in the future, and only wake a node up when it is going to contact another node. A node stays asleep during inter-contact times, and probes for the predicted contact with the optimal interval [22] after it wakes up.

TABLE II

TRACE SUMMARY

\begin{tabular}{c|ccc}
\hline Trace & MIT Reality & UCSD & Infocom \\
\hline \hline Network type & Bluetooth & WiFi & Bluetooth \\
Number of devices & 97 & 275 & 78 \\
Number of internal contacts & 114,046 & 123,225 & 182,951 \\
Duration (days) & 246 & 77 & 4 \\
Period of contact probing (secs) & 120 & 20 & 120 \\
Avg. contact duration (hours) & 0.57 & 5.45 & 0.142 \\
Avg. pairwise inter-contact & 84.13 & 47.17 & 1.883 \\
time (hours) & & & \\
\hline
\end{tabular}

\section{B. Network Model}

Opportunistic contacts among nodes are described by a network contact graph $G(V, E)$, where stochastic contact process between a node pair $i, j \in V$ is modeled as an edge $e_{i j} \in E$. We assume that node contacts are symmetric and $G$ 


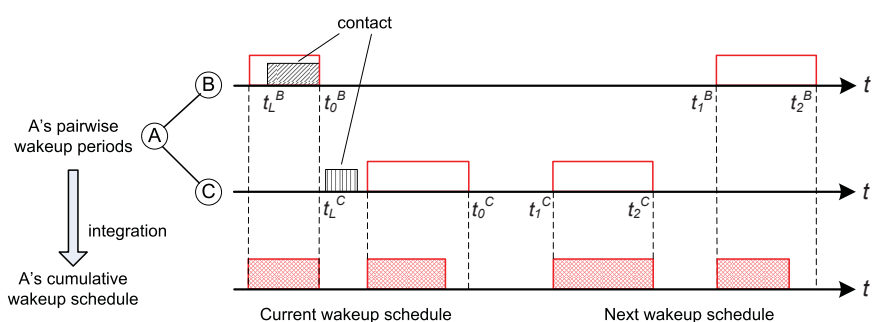

Fig. 2. Wakeup scheduling at a node $A$. $A$ independently schedules pairwise wakeup periods with its contacted neighbors $B$ and $C$, and then integrates these pairwise wakeup periods to generate its cumulative wakeup schedule.

is hence undirected. The characteristics of an edge $e_{i j} \in E$ are mainly determined by the properties of inter-contact time among nodes. Similar to previous work [1], [12], we consider the pairwise node inter-contact time as exponentially distributed, which has been validated from both theoretical [3] and experimental aspects [25]. Contacts between nodes $i$ and $j$ then form a Poisson process with contact rate $\lambda_{i j}$, which is calculated in real time from the cumulative contacts between nodes $i$ and $j$. In the rest of this paper, we call a pair of nodes $i, j$ as contacted neighbors if $\lambda_{i j}>0$, and call the node set $\left\{j \mid \lambda_{i j}>0\right\} \subseteq V$ as the contacted neighborhood of node $i$.

\section{The Big Picture}

We consider a generic scenario for opportunistic communication, in which a node $A$ carrying data as relay independently determines whether to forward data to another node $B$ when $A$ opportunistically contacts $B$. The goal of our wakeup scheduling is hence to ensure that both nodes $A$ and $B$ stay awake each time when they contact each other.

Being different from current schemes [23], [4] in which nodes' wakeup schedules are predetermined with fixed periods, we propose to adaptively schedule wakeup periods of mobile nodes in real time. As illustrated in Figure 2, a pair of contacted neighbors $A$ and $B$ independently schedule their next pairwise wakeup period $\left[t_{1}, t_{2}\right]$ when their current pairwise wakeup period ends at time $t_{0}$, to minimize the energy consumption during $\left[t_{0}, t_{2}\right]$ while ensuring that their next contact happens during $\left[t_{1}, t_{2}\right]$ with high probability.

In our problem formulation, energy consumption during $\left[t_{1}, t_{2}\right]$ is measured by the active ratio $r=\frac{t_{2}-t_{1}}{t_{2}-t_{0}}$. We focus on minimizing $r$ with the performance requirement of opportunistic communication specified by

$\mathbb{P}\left(t_{1}-t_{L} \leq t_{A B} \leq t_{2}-t_{L}\right) \geq p \cdot \mathbb{P}\left(t_{0}-t_{L}<t_{A B} \leq t_{2}-t_{L}\right)$,

where $t_{A B}$ is the random variable indicating the next intercontact time between $A$ and $B$, and $t_{L}$ is the last time when $A$ and $B$ contacted each other. This constraint, controlled by the parameter $p$, specifies the requirement of probability for $A$ to contact $B$ during $\left[t_{1}, t_{2}\right]$, with respect to that probability during $\left[t_{0}, t_{2}\right]$. In applications requiring high communication performance, a larger value of $p$ can be applied to ensure sufficient contact opportunities. In contrast, in scenarios where nodes' battery life is very limited, the value of $p$ can be reduced to save more energy.
Our basic idea is to probabilistically predict the next contact happened after $t_{0}$ based on contact records in the past. With the prediction results, we formally optimize the pairwise wakeup period for minimizing $r$, while satisfying the requirement in Eq. (1). As shown in Figure 2, after the predicted contact with $B$ happened during the current wakeup period, $A$ finishes its current pairwise wakeup period with $B$ and schedules the next immediately if either of the following two conditions is satisfied: (i) data transmission with $B$ completes, and (ii) contact with $B$ has finished. On the other hand, $A$ 's contact with $C$ is missed by its current pairwise wakeup period with $C$. We develop techniques to handle such inaccuracy of contact prediction when scheduling $A$ 's next pairwise wakeup period with $C$, to avoid missing contacts with $C$ again in the future.

Afterwards, $A$ integrates all its scheduled pairwise wakeup periods in a cascaded manner, to generate its cumulative wakeup schedule. Figure 2 shows that the cumulative active ratio of $A$ can be further improved during such integration, due to the coexistence of multiple pairwise wakeup periods. For example, $A$ 's next pairwise wakeup period with $B$ is shortened when being integrated into $A$ 's cumulative wakeup schedule, because it is also possible for $A$ to contact $B$ during its next pairwise wakeup period with $C$, which has been scheduled earlier. We also propose techniques to preserve scheduling consistency by effectively coordinating multiple nodes. By doing this, we ensure that any two contacted neighbors stay awake simultaneously when they contact each other.

\section{Pairwise Wakeup Scheduling}

In this section, we determine the optimal wakeup period for a pair of contacted neighbors to minimize their active ratio $r$ with respect to the performance requirement in Eq. (1). Without loss of generality, Eq. (1) is satisfied if

$$
\begin{aligned}
& \mathbb{P}\left(t_{1}-t_{L} \leq t_{A B} \leq t_{2}-t_{L}\right) \\
& \geq p \cdot\left(1-\mathbb{P}\left(0<t_{A B} \leq t_{0}-t_{L}\right)\right)=p_{s} .
\end{aligned}
$$

According to the problem formulation in Section III-C, the quantities $t_{0}$ and $t_{L}$ are known and fixed when scheduling the next pairwise wakeup period of a pair of contacted neighbors $A$ and $B$. As a result, Eq. (2) will be used as the performance requirement in this section for simplicity.

\section{A. Optimizing Pairwise Wakeup period}

We schedule the next pairwise wakeup period of $A$ and $B$ by predicting their next contact in the future, i.e., estimating their next inter-contact time. According to our network modeling in Section III-B, the pairwise inter-contact time between $A$ and $B$ is exponentially distributed. Hence, we have

$$
p_{s}=p \cdot\left(1-\int_{0}^{t_{0}-t_{L}} \lambda e^{-\lambda t} d t\right)=p \cdot e^{-\lambda\left(t_{0}-t_{L}\right)},
$$

where $\lambda$ is the pairwise contact rate between $A$ and $B$.

Letting the length of the next pairwise wakeup period be $\Delta=t_{2}-t_{1}$, we have the active ratio $r=\frac{t_{2}-t_{1}}{t_{2}-t_{0}}=\frac{\Delta}{t_{1}-t_{0}+\Delta}$. As a result, Eq. (2) can be rewritten as 


$$
\begin{aligned}
& \mathbb{P}\left(t_{1}-t_{L} \leq t_{A B} \leq t_{2}-t_{L}\right)=\int_{t_{1}-t_{L}}^{t_{2}-t_{L}} \lambda e^{-\lambda t} d t \\
& =e^{-\lambda\left(t_{1}-t_{L}\right)}-e^{-\lambda\left(t_{1}+\Delta-t_{L}\right)} \geq p_{s},
\end{aligned}
$$

which means

$$
\Delta_{\min }=\frac{1}{\lambda} \cdot \ln \frac{1}{1-p_{s} e^{\lambda\left(t_{1}-t_{L}\right)}}
$$

is the minimum value of $\Delta$ satisfying Eq. (4), given a fixed value of $t_{1} \in\left[t_{0}, t_{L}+\frac{1}{\lambda} \ln \frac{1}{p_{s}}\right)$.

The major focus for optimizing the pairwise wakeup period is then to determine the optimal value of $t_{1}$, which estimates the time that the next contact between $A$ and $B$ is likely to happen. Letting

we have

$$
\frac{1}{r_{\min }}=1+\frac{t_{1}-t_{0}}{\Delta_{\min }}=1+\frac{\lambda\left(t_{1}-t_{0}\right)}{\ln \frac{1}{1-p_{s} e^{\lambda\left(t_{1}-t_{L}\right)}}}
$$

$$
\begin{aligned}
\frac{\partial\left(1 / r_{\min }\right)}{\partial t_{1}}= & \frac{\lambda}{\ln \frac{1}{1-p_{s} e^{\lambda\left(t_{1}-t_{L}\right)}}} \\
& -\frac{p_{s} \lambda^{2}\left(t_{1}-t_{0}\right) e^{\lambda\left(t_{1}-t_{L}\right)}}{\left(1-p_{s} e^{\lambda\left(t_{1}-t_{L}\right)}\right) \cdot\left(\ln \frac{1}{1-p_{s} e^{\lambda\left(t_{1}-t_{L}\right)}}\right)^{2}},
\end{aligned}
$$

which enables the following formal analysis.

Lemma 1: $\left.\frac{\partial\left(1 / r_{\min }\right)}{\partial t_{1}}\right|_{t_{1}=t_{0}}>0,\left.\frac{\partial\left(1 / r_{\min }\right)}{\partial t_{1}}\right|_{t_{1} \rightarrow t_{L}+\frac{1}{\lambda} \ln \frac{1}{p_{s}}}=$ $-\infty$.

Proof: First, from Eq. (6) we immediately have

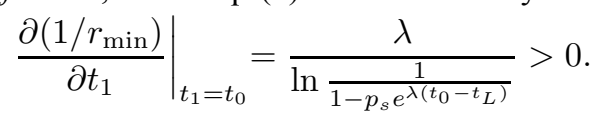

Second, it is easy to see that

$$
\left.\ln \frac{1}{1-p_{s} e^{\lambda\left(t_{1}-t_{L}\right)}}\right|_{t_{1} \rightarrow t_{L}+\frac{1}{\lambda} \ln \frac{1}{p_{s}}}=\ln \frac{1}{0}=\infty .
$$

Letting $x=1-p_{s} e^{\lambda\left(t_{1}-t_{L}\right)}$, we have

$$
\lim _{x \rightarrow 0}\left(x \cdot\left(\ln \frac{1}{x}\right)^{2}\right)=0,
$$

and hence

$$
\lim _{t_{1} \rightarrow t_{L}+\frac{1}{\lambda} \ln \frac{1}{p_{s}}} \frac{p_{s} \lambda^{2}\left(t_{1}-t_{0}\right) e^{\lambda\left(t_{1}-t_{L}\right)}}{\left(1-p_{s} e^{\lambda\left(t_{1}-t_{L}\right)}\right) \cdot\left(\ln \frac{1}{1-p_{s} e^{\lambda\left(t_{1}-t_{L}\right)}}\right)^{2}}=\infty
$$

from which the lemma follows.

From Lemma 1, we furthermore have the following lemma:

Lemma 2: The equation $f\left(t_{1}\right)=\frac{\partial\left(1 / r_{\min }\right)}{\partial t_{1}}=0$ has and only has one real solution with $t_{1} \in\left[t_{0}, t_{L}+\frac{1}{\lambda} \ln \frac{1}{p_{s}}\right)$.

Proof: Based on Lemma 1, the proof of Lemma 2 is equivalent to prove that the value of $f\left(t_{1}\right)$ is monotonically decreasing when $t_{1} \in\left[t_{0}, t_{L}+\frac{1}{\lambda} \ln \frac{1}{p_{s}}\right]$.

Being similar with the proof of Lemma 1, letting $x=1-$ $p_{s} e^{\lambda\left(t_{1}-t_{L}\right)}$, we immediately have $\frac{\partial x}{\partial t_{1}}<0$ for $\forall t_{1} \in \mathbb{R}$. As a result, Lemma 2 is proved by considering

and

$$
\frac{\partial}{\partial x}\left(1 / \ln \frac{1}{x}\right)>0
$$

$$
\frac{\partial}{\partial x}\left(x \cdot \ln \left(\frac{1}{x}\right)^{2}\right)>0 \text {. }
$$

With these two lemmas, we have the following theorem:

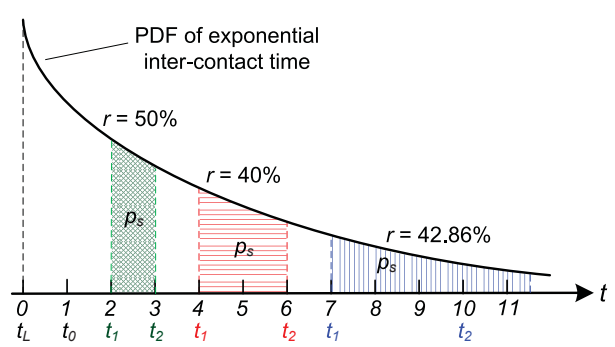

Fig. 3. Illustration of minimizing the active ratio $r$ with the optimal value of $t_{1}$. When $t_{1}=2$ being close to $t_{0}$, we have $\Delta=1$ and $r=1 / 2=50 \%$. When $t_{1}$ increases to $4, \Delta$ increases to 2 accordingly to satisfy the required contact probability $p_{s}$, which reduces $r$ to $2 / 5=40 \%$. In contrast, $\Delta$ is enlarged to 4.5 when $t_{1}=7$, and increases $r$ to $4.5 / 10.5=42.86 \%$.

Theorem 1: The active ratio $r$ is globally minimized when $f\left(t_{1}^{*}\right)=\left.\frac{\partial}{\partial t_{1}}\left(\frac{1}{r_{\min }}\right)\right|_{t_{1}=t_{1}^{*}}=0$.

An intuitive explanation about Theorem 1 is illustrated in Figure 3 . When $t_{1}$ increases with $\Delta$ unchanged, the active ratio $r$ decreases. However, since the Probability Density Function (PDF) of exponential distribution is $p(t)=\lambda e^{-\lambda t}$ which is monotonically decreasing with $t$, a larger value of $\Delta$ is needed to satisfy Eq. (2), which increases $r$. The value of $r$ is hence minimized when the value of $t_{1}$ is optimized.

From Eq. (6), the following equation needs to be solved to find out the optimal value of $t_{1}$ :

$$
\lambda\left(t_{1}-t_{0}\right)=\frac{1-p_{s} e^{\lambda\left(t_{1}-t_{L}\right)}}{p_{s} e^{\lambda\left(t_{1}-t_{L}\right)}} \cdot \ln \frac{1}{1-p_{s} e^{\lambda\left(t_{1}-t_{L}\right)}} .
$$

In practice, Eq. (7) can be efficiently solved by numerical iterations, and the optimal value of $t_{1}$ is then used in Eq. (5) to calculate the length $(\Delta)$ of pairwise wakeup period.

\section{B. Handling Inaccuracy of Contact Prediction}

The accuracy of our contact prediction is subject to the randomness of mobile node behaviors. The predicted contact may not happen during the current wakeup period, indicating inaccurate estimation of inter-contact time. In this case, the time $t_{L}$ when the missed contact happened, which is required for scheduling the next wakeup period as described in Section IV-A, is unknown. Moreover, such inaccuracy complicates the scheduling of the next wakeup period, because the prediction error will impact the estimation of the next inter-contact time.

Our basic idea to handle such prediction inaccuracy is to schedule the next wakeup period to start earlier and last longer, so as to ensure that the next predicted contact will not be missed again. As illustrated in Figure 4, the area of $S_{1}$ is much larger than that of $S_{2}$, indicating that the majority of missed probability mass is located before the current wakeup period which starts at $t_{s}$. Hence, we believe that the missed contact most probably happened before $t_{s}$, and the next contact will also happen earlier than we expect.

The wakeup scheduling scheme presented in Section IV-A can be described as a function $\left\{t_{1}, \Delta\right\}=f\left(t_{0}, t_{L}, p, \lambda\right)$, which calculates $t_{1}$ by solving Eq. (7) and then $\Delta$ from Eq. (5). When a predicted contact does not happen during the current wakeup period, the next wakeup period is scheduled differently as

$$
\left\{t_{1}^{\prime}, \Delta^{\prime}\right\}=f\left(t_{s}, t_{s}-d_{\max }, p, \lambda\right),
$$




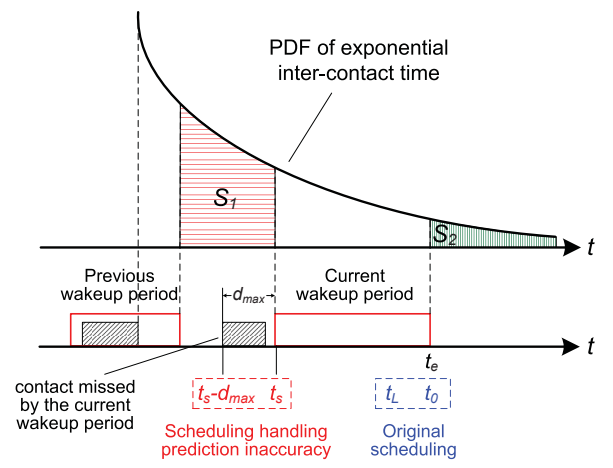

Fig. 4. Handling inaccuracy of contact prediction. The missed contact is considered to happen before the current wakeup period started at $t_{s}$.

where $d_{\max }$ is the maximum contact duration among all the recorded pairwise contacts in the past.

This scheduling method is illustrated in Figure 4, in which we consider that the current wakeup period should have started earlier, and use $t_{s}-d_{\max }$ to estimate when the missed contact happened before $t_{s}$. The characteristics of such scheduling is formally analyzed by the following theorem.

Theorem 2: Letting $\left\{t_{1}, \Delta\right\}=f\left(t_{0}, t_{L}, p, \lambda\right)$, where $t_{0}=$ $t_{e}$ is the time when the current wakeup period ends, we have $t_{1}^{\prime}<t_{1}$ and $\Delta^{\prime}>\Delta$ for $\forall t_{L} \in\left(t_{s}, t_{e}\right)$, where $t_{1}^{\prime}$ and $\Delta^{\prime}$ are calculated according to Eq. (8).

Proof: Letting $\delta_{1}=t_{e}-t_{s}, \delta_{2}=t_{L}-\left(t_{s}-d_{\max }\right)$.

(1) When $t_{0}$ decreases, the 1.h.s. of Eq. (7) increases, and hence $t_{1}$ decreases accordingly. Similarly, $t_{1}$ decreases when $t_{L}$ decreases because

$$
\frac{\partial}{\partial\left(t_{1}-t_{L}\right)}\left(\frac{1-p_{s} e^{\lambda\left(t_{1}-t_{L}\right)}}{p_{s} e^{\lambda\left(t_{1}-t_{L}\right)}} \cdot \ln \frac{1}{1-p_{s} e^{\lambda\left(t_{1}-t_{L}\right)}}\right)<0,
$$

for $\forall t_{1} \in\left[t_{0}, t_{L}+\frac{1}{\lambda} \ln \frac{1}{p_{s}}\right)$.

Hence we have $t_{1}^{\prime}<t_{1}$ considering that $\delta_{1}>0$ and $\delta_{2}>0$.

(2) Letting $t_{1}^{\prime}=t_{1}-\epsilon_{1}$ and $t_{L}^{\prime}=t_{L}-\epsilon_{L}$ with $t_{L}^{\prime}=t_{s}-d_{\max }$ and $\epsilon_{1}, \epsilon_{L}>0$, in Eq. (7) we have

$$
\begin{aligned}
& \lambda\left(t_{1}-\epsilon_{1}-t_{s}\right)= \\
& \frac{1-p_{s} e^{\lambda\left(t_{1}-t_{L}+\epsilon_{L}-\epsilon_{1}\right)}}{p_{s} e^{\lambda\left(t_{1}-t_{L}+\epsilon_{L}-\epsilon_{1}\right)}} \cdot \ln \frac{1}{1-p_{s} e^{\lambda\left(t_{1}-t_{L}+\epsilon_{L}-\epsilon_{1}\right)}} .
\end{aligned}
$$

From Eq. (9) we know that $\epsilon_{L}-\epsilon_{1}>0$ and hence $t_{1}^{\prime}-t_{L}^{\prime}>$ $t_{1}-t_{L}$. This indicates that $\Delta^{\prime}>\Delta$ according to Eq. (5).

Essentially, Theorem 2 shows that the next wakeup period will start earlier and last longer. Our method hence minimizes the chance for the predicted contacts to be missed again in the future. The length of wakeup period will be resumed normal as soon as a predicted contact happens during the wakeup period.

\section{Cumulative Wakeup Scheduling}

A node may have multiple contacted neighbors on the network contact graph. In this section, we integrate the pairwise wakeup periods of a node being scheduled with all its contacted neighbors together, to generate the cumulative wakeup schedule for this node. We also propose techniques to effectively maintain the scheduling consistency among different nodes during such integration.

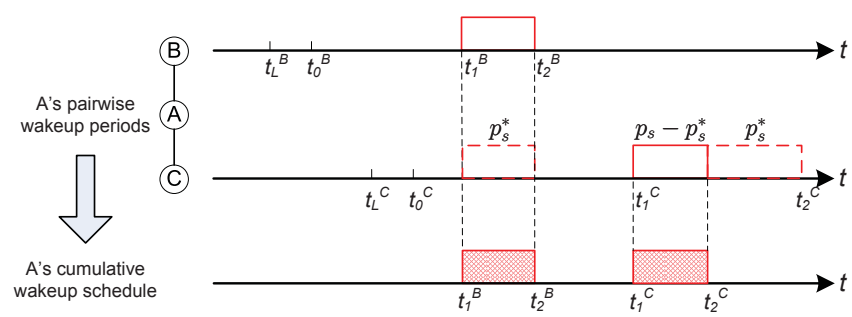

Fig. 5. Motivation of optimizing the cumulative active ratio due to the coexistence of multiple pairwise wakeup periods

\section{A. Cascaded Integration of Pairwise Wakeup Schedules}

The cumulative wakeup schedule of a node is generated by integrating its pairwise wakeup periods with all its contacted neighbors in a cascaded manner in real time. As illustrated in Figure 5, when $A$ contacts its contacted neighbor $C$ at time $t_{0}^{C}$, it integrates its next pairwise wakeup period $\mathbb{L}_{C}=\left[t_{1}^{C}, t_{2}^{C}\right]$ with $C$ together with the existing pairwise wakeup period $\mathbb{L}_{B}=\left[t_{1}^{B}, t_{2}^{B}\right]$ with $B$. In this way, $A$ integrates the pairwise wakeup period with each of its contacted neighbors into its cumulative wakeup schedule when it contacts that neighbor.

We notice that the cumulative active ratio of a node can be further optimized due to the coexistence of multiple pairwise wakeup periods of this node with its contacted neighbors. For example in Figure 5, when $A$ schedules $\mathbb{L}_{C}$ at time $t_{0}^{C}$, the length of $\mathbb{L}_{C}$ could be reduced because $A$ also has the probability $p_{s}^{*}$ to contact $C$ during $\mathbb{L}_{B}$ which has been scheduled earlier. We adaptively optimize the scheduling of each pairwise wakeup period considering other existing pairwise wakeup periods which have already been scheduled. When a node $A$ schedules its pairwise wakeup period with $C$ at time $t_{0}^{C}$, its probability for calculating $t_{1}$ and $\Delta$ in Eqs. (5) and (7) is reduced from $p_{s}$ to $p_{s}-p_{s}^{*}$, such that

$$
p_{s}^{*}=\sum_{i=1}^{k}\left(e^{-\lambda_{A C}\left(t_{1}^{i}-t_{L}\right)}-e^{-\lambda_{A C}\left(t_{2}^{i}-t_{L}\right)}\right),
$$

where $\lambda_{A C}$ is the pairwise contact rate between $A$ and $C$, and each $\mathbb{L}^{i}=\left[t_{1}^{i}, t_{2}^{i}\right]$ corresponds to an existing pairwise wakeup period with $t_{1}^{i}>t_{0}^{C}$. We merge multiple pairwise wakeup periods which overlap with each other as one $\mathbb{L}^{i}$ in Eq. (10). In particular, if $p_{s}^{*} \geq p_{s}$, a pairwise wakeup period between $A$ and $C$ is not needed at $t_{0}^{C}$. Instead, $A$ will set $\max \left\{t_{2}^{i}\right\}$ as the next time to schedule its pairwise wakeup period with $C$.

The following theorem shows that the newly scheduled pairwise wakeup period of $A$ with $C$ after such optimization will start later and last shorter.

Theorem 3: Letting $\left\{t_{1}, \Delta\right\}=f\left(t_{0}, t_{L}, p, \lambda\right)$ and $\left\{t_{1}^{*}, \Delta^{*}\right\}=f\left(t_{0}, t_{L}, p-p^{*}, \lambda\right)$, we have $t_{1}^{*}>t_{1}$ and $\Delta^{*}<\Delta$ for $\forall p^{*}>0$.

Proof: From Eq. (3) we know that

$$
p_{s}^{*}=\left(p-p^{*}\right) \cdot\left(1-e^{-\lambda\left(t_{0}-t_{L}\right)}\right)<p_{s} .
$$

(1) For simplicity we rewrite Eq. (7) as $\lambda\left(t_{1}-t_{0}\right)=$ $g\left(t_{1}, t_{L}, p_{s}, \lambda\right)$. If $t_{1}^{*} \leq t_{1}$, based on Eq. (9) we have

$$
g\left(t_{1}^{*}, t_{L}, p^{*}, \lambda\right)>g\left(t_{1}, t_{L}, p, \lambda\right)
$$

which contradicts with $\lambda\left(t_{1}^{*}-t_{0}\right) \leq \lambda\left(t_{1}-t_{0}\right)$. Hence $t_{1}^{*}>t_{1}$.

(2) From Eq. (9), letting $x=p_{s} e^{\lambda\left(t_{1}-t_{L}\right)}$ we have 


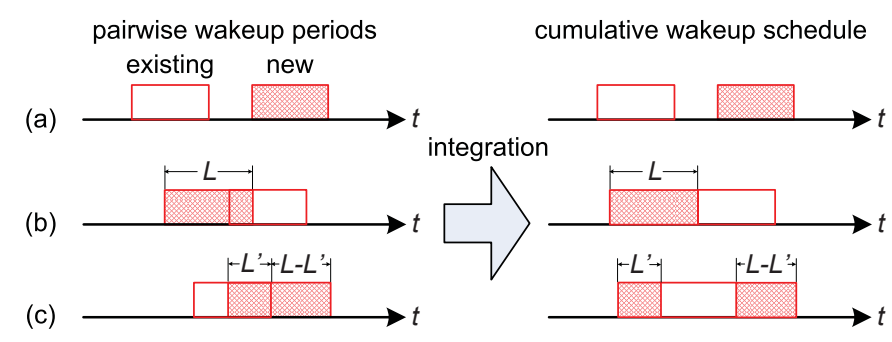

Fig. 6. Integration of pairwise wakeup periods. If pairwise wakeup periods overlap, the newly scheduled wakeup period will be changed to happen earlier.

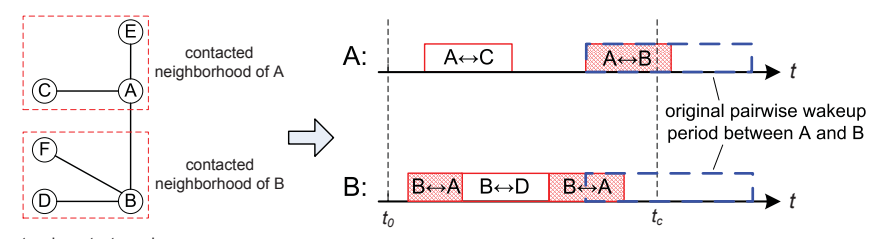

network contact graph

Fig. 7. Illustration of scheduling inconsistency. When $A$ actually contacts $B$ at time $t_{c}, B$ has already been asleep and the contact is missed.

$$
\frac{\partial}{\partial x}\left(\frac{x}{1-x} \ln \frac{1}{x}\right)<0,
$$

which shows that

$$
p_{s}^{*} e^{\lambda\left(t_{1}^{*}-t_{L}\right)}<p_{s} e^{\lambda\left(t_{1}-t_{L}\right)} .
$$

We then have $\Delta^{*}<\Delta$ by applying Eq. (12) to Eq. (5).

After the new pairwise wakeup period has been scheduled based on $p_{s}-p_{s}^{*}$, it will be integrated with other existing pairwise wakeup periods as illustrated in Figure 6 . When the newly scheduled wakeup period overlaps with other existing wakeup periods, the new wakeup period will be changed to happen earlier, as shown in Figure 6(b). In particular, if the new wakeup period happens later than an existing one, the new wakeup period will be split as shown in Figure 6(c).

\section{B. Preserving Scheduling Consistency}

As shown in Figure 7, nodes $A$ and $B$ may have different contacted neighborhoods on the network contact graph. Due to this difference, integrating pairwise wakeup periods of a node as described in Section V-A may lead to inconsistency among the wakeup schedules of contacted neighbors. For example in Figure 7 , nodes $A$ and $B$, which are contacted neighbors, optimize their pairwise wakeup period with each other in different ways due to the difference of their contacted neighborhood. As a result, when $A$ actually contacts $B$ at time $t_{c}, B$ has already been asleep and the contact is missed.

Our basic idea to address such scheduling inconsistency is to let two contacted neighbors communicate with each other at $t_{0}$ before they optimize their next pairwise wakeup period, so as to ensure that they have identical pairwise wakeup period with each other and still satisfy the requirement of contact probability. More specifically, two contacted neighbors $A$ and $B$ exchange the information about their existing wakeup schedules, and furthermore schedule their next pairwise wakeup period based on this common information in two steps.

First, as illustrated in Figure 8(a), the following probability

$$
p_{s}^{*}=\int_{t_{1}^{B D}-t_{L}^{A B}}^{t_{2}^{A C}-t_{L}^{A B}} e^{-\lambda_{A B} t} d t,
$$

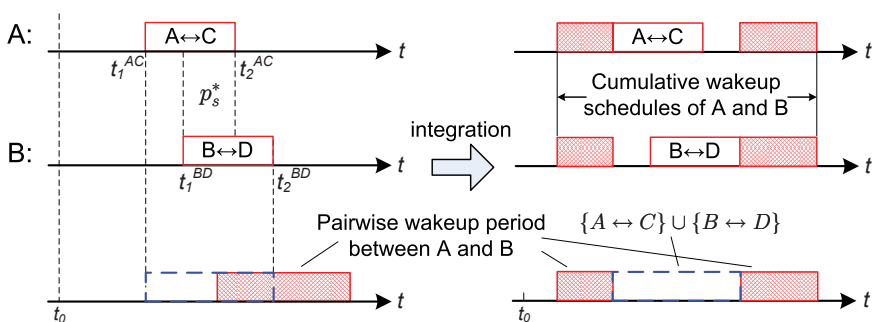

(a) before integration

(b) after integration

Fig. 8. Preserving scheduling consistency. The pairwise wakeup period satisfies the requirement of contact probability at both $A$ and $B$.

where $t_{L}^{A B}$ is the last time when $A$ contacted $B$, is used to calculate the next pairwise wakeup period between $A$ and $B$ as suggested in Section V-A. Since both $A$ and $B$ are awake during $\left[t_{1}^{B D}, t_{2}^{A C}\right]$, the scheduled pairwise wakeup period satisfies the requirement of contact probability at both $A$ and $B$. In general, $p_{s}^{*}$ is calculated based on all the overlapping periods of the existing wakeup schedules of $A$ and $B$.

Second, the pairwise wakeup period between $A$ and $B$ scheduled in the first step may overlap with other existing wakeup periods at $A$ or $B$. In this case, we get the union of the existing wakeup schedules at $A$ and $B$, and then integrate the pairwise wakeup period between $A$ and $B$ with such union as described by Figure 6 . The cumulative wakeup schedules of $A$ and $B$ after integration are illustrated in Figure 8(b).

\section{Performance Evaluations}

We compare the performance of our proposed scheme on improving the energy efficiency of opportunistic communication, with the following schemes which reduce energy consumption by optimizing the interval of contact probing:

- No Energy Saving: the interval of contact probing is not optimized, and the default intervals of contact probing listed in Table II are used in all experiments.

- Low-Duty-Cycle [23]: each node runs a fixed-length duty cycle, which optimizes the interval of contact probing but is determined a priori according to the network conditions and performance requirements.

- Cyclic Difference Set (CDS) [4]: duty cycle of each node consists of several slots. The active slots are scheduled according to the predetermined CDS settings, to ensure that different nodes stay awake simultaneously during specific active slots while minimizing the active ratio.

- Adaptive Probing [22]: the probing interval is adaptively optimized according to the time-varying contact rates.

\section{A. Simulation Setup}

We evaluate the energy efficiency of forwarding data to a specific destination, based on traces listed in Table II. We first randomize the data generation time and lifetime, and then randomly pick the data sources and destinations among the nodes with non-zero contact counts during data lifetime. In our experiments, data is forwarded by the following strategies with betweenness as the forwarding metric [15]:

- Compare-and-Forward [5]: a relay replicates data to other nodes with higher metrics than that of itself. 


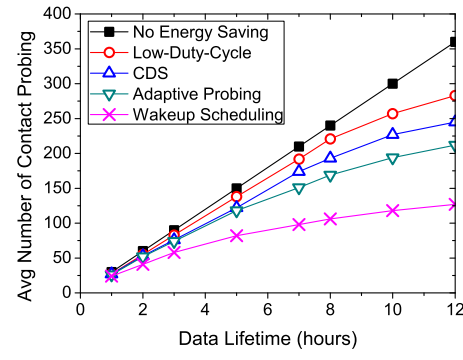

(a) Energy consumption

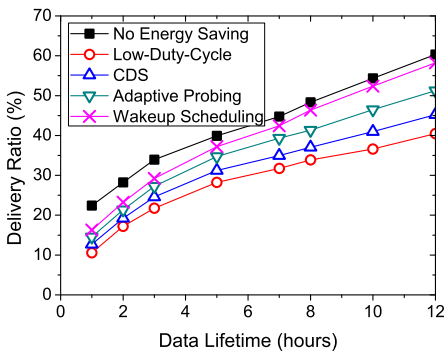

(b) Delivery ratio

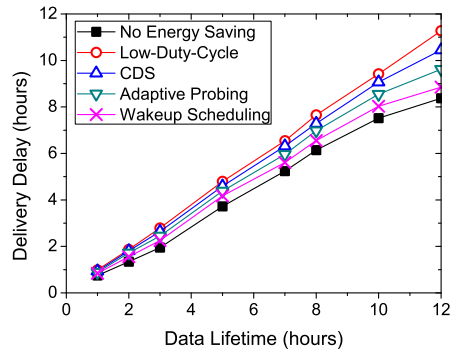

(c) Delivery delay

Fig. 9. Energy efficiency of opportunistic communication with different data lifetime. Compare-and-Forward strategy is used in the Infocom trace.

- Delegation [8]: the number of data replicas in the network is reduced, because a relay only replicates data to nodes with higher metrics than any existing relay.

- Spray-and-Focus [21]: the maximum number $(N)$ of data replicas is fixed. Each relay forwards data following Compare-and-Forward without retaining a local copy.

We evaluate the performance of data forwarding in data delivery ratio and delay, and measure the energy efficiency of opportunistic communication using the average number of contact probing per node during data lifetime. We assume that all the data is transmitted via Bluetooth with channel bandwidth of $1 \mathrm{Mbps}$, and the size of each data is $100 \mathrm{Mb}$. We assume that each node has sufficient buffer to store the data. Each experiment is repeated 500 times with random data sources and destinations for statistical convergence.

\section{B. Energy Efficiency of Opportunistic Communication}

In this section, we evaluate the performance of our proposed scheme on improving the energy efficiency of opportunistic communication using the Infocom trace. We first set the parameter $p$ in our scheme to be 0.7 , and apply different energy saving schemes to the Compare-and-Forward Strategy. The results with different data lifetime $(T)$ are shown in Figure 9. Our proposed wakeup scheduling scheme dramatically reduces the energy consumption of opportunistic communication. As shown in Figure 9(a), when $T$ is 12 hours, our scheme reduces the average number of contact probing per node by $65 \%$ compared to that without energy saving, and performs over $30 \%$ better than the Adaptive Probing scheme does. The major reason for such difference is that our scheme enables the nodes to remain asleep during the long inter-contact times, so as to avoid a large amount of unnecessary contact probing. Note that in Figure 9(a), when $T$ increases, more energy is consumed in opportunistic communication, because more contact probing is performed during data lifetime. In these cases, our scheme also saves a higher percentage of energy, because a larger portion of data lifetime corresponds to inter-contact time periods.

Meanwhile, our proposed scheme effectively preserves the performance of data forwarding, and hence improves the energy efficiency of opportunistic communication. As shown in Figures 9(b) and 9(c), the performance of data forwarding is mainly constrained by $T$. When $T$ increases from 1 hour to 12 hours, the data delivery ratio increases from $20 \%$ to $60 \%$ for No Energy Saving. When our proposed scheme is applied for energy saving, Figure 9(b) shows that the data delivery ratio is

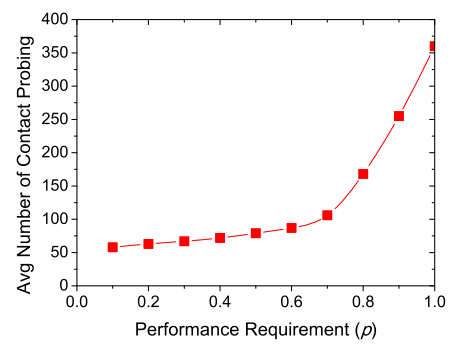

(a) Energy consumption

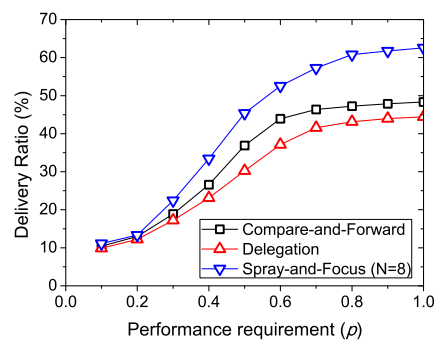

(b) Delivery ratio
Fig. 10. Energy efficiency of opportunistic communication with different different performance requirements, specified by the value of parameter $p$.

slightly reduced by less than $10 \%$. Comparatively, Low-DutyCycle reduces the delivery ratio by over $35 \%$, because the predetermined and fixed duty cycles lead to higher chances of missing contacts. Adaptive Probing and CDS work better, but also have noticeable performance degradation because they did not intentionally predict node contacts in the future. Similar cases apply to data delivery delay as shown in Figure 9(c).

We also evaluate our scheme with different performance requirements, specified by the parameter $p$. The evaluation results with different data forwarding strategies are shown in Figure 10, where $T=8$ hours and $N=8$ for Spray-and-Focus. Figure 10(a) shows that the amount of energy consumption increases slowly when $p<0.6$, but increases much faster when $p \geq 0.7$. Figure 10 (b) shows that the data delivery ratio cannot be noticeably improved by further increasing the value of $p$ when $p \geq 0.7$, but will be seriously degraded when $p \leq 0.4$.

These results indicate that appropriately determining the value of $p$ is critical to the energy efficiency of opportunistic communication. When $p$ is large, further increasing $p$ will not help improve the communication performance, but simply prevents the wasted energy from being saved. In contrast, a small value of $p$ makes nodes remain asleep during most of the time. As a result, many contact opportunities are missed and the communication performance is seriously impaired. From Figure 10, we conclude that the value of $p$ should be in the range of $[0.4,0.7]$ for the Infocom trace. In practice, the value of $p$ should be adaptively determined according to the specific network scenarios and application requirements.

\section{Accuracy of Contact Prediction}

In this section, we evaluate the accuracy of our contact prediction method presented in Section IV. As described in Section III-C, our basic idea for wakeup scheduling is to prob- 

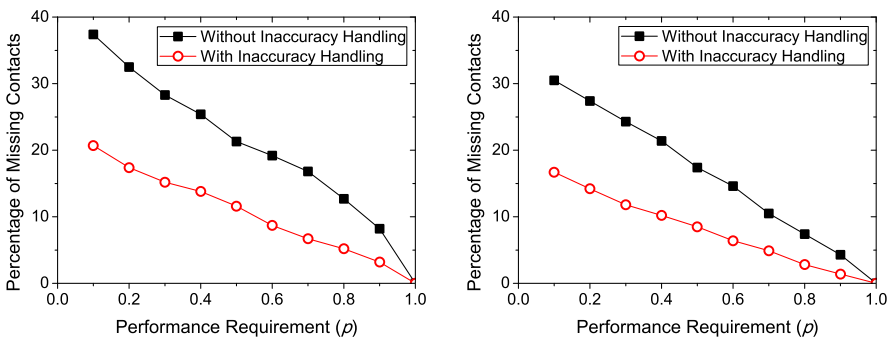

(a) Among all contacts

(b) Among contacts between relays

Fig. 11. Evaluating the accuracy of contact prediction using the percentage of missing contacts due to wakeup scheduling.

abilistically predict node contacts in the future. The accuracy of contact prediction, therefore, is crucial to improving the energy efficiency of opportunistic communication.

We first evaluate the accuracy of contact prediction using the percentage of missing contacts due to wakeup scheduling. The results on the Infocom trace, with different values of $p$, are shown in Figure 11. When the value of $p$ is small, nodes remain asleep during most of time, and our scheme has higher chances to miss contacts. As shown in Figure 11(a), more than $25 \%$ of contacts are missed when $p<0.4$, leading to serious degradation of communication performance shown in Figure 10(b). Nevertheless, Figure 11(a) also shows that such inaccuracy of contact prediction quickly diminishes when $p$ increases, and the percentage of missing contacts is reduced to $10 \%$ when $p=0.8$. This inaccuracy can also be effectively handled by our techniques proposed in Section IV-B. By enlarging the pairwise wakeup periods and scheduling them to be happening earlier, we control the percentage of missing contacts to be lower than $10 \%$ for $p \geq 0.5$.

In particular, in Figure 11(b) we evaluate the percentage of missed contacts among the contacts between relays, which could actually be used for forwarding data. As shown in Figure 11(b), such percentage is generally $10 \%$ lower than that in Figure 11(a), and is even as low as 5\% when $p>0.6$. The major reason for such difference is that the relays generally have higher chances to contact other nodes when betweenness is used as the forwarding metric. The inter-contact times of relays, which are hence shorter than other nodes, lead to higher accuracy of contact prediction.

Our proposed inaccuracy handling techniques ensure high accuracy of contact prediction, and further ensure that the performance of opportunistic communication can be effectively preserved. As shown in Figure 12, when $p=0.7$, our inaccuracy handling techniques improve the data delivery ratio by up to $15 \%$, and also reduce the delivery delay. In particular, when $T$ is shorter, a contact between relays has a higher chance to be missed, and our inaccuracy handling techniques are more effective on improving data delivery ratio.

\section{Impacts of Cumulative Wakeup Scheduling}

We evaluate the impacts of cumulative wakeup scheduling proposed in Section V on energy efficiency of opportunistic communication. The pairwise wakeup periods of a node is integrated in a cascaded manner to generate its cumulative

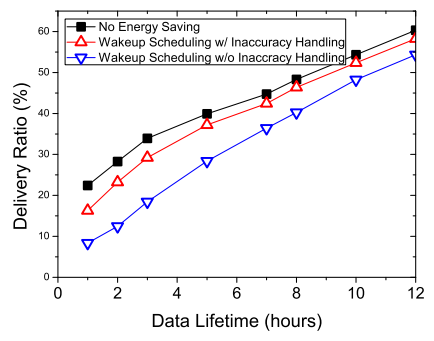

(a) Delivery ratio

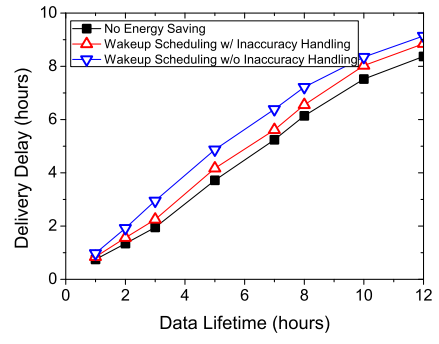

(b) Delivery delay
Fig. 12. The impact of handling inaccuracy of contact prediction on preserving the performance of data forwarding. Compare-and-Forward strategy is used in the Infocom trace.

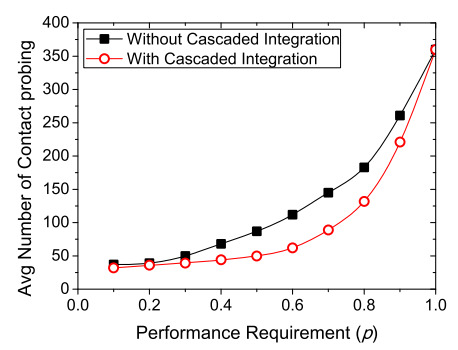

(a) Energy consumption

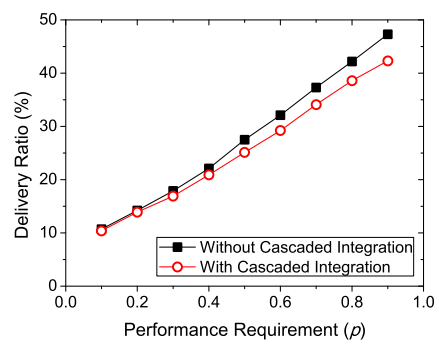

(b) Delivery ratio
Fig. 13. Evaluating the impacts of cumulative wakeup scheduling. Delegation strategy is used in the MIT Reality trace.

wakeup schedule, which is further optimized due to the coexistence of pairwise wakeup periods. For comparison, we simply combine the pairwise wakeup periods of a node together without cascaded integration, and the comparison results are shown in Figure 13, where we perform experiments using the MIT Reality trace and setting data lifetime as $T=1$ week.

Figure 13(a) shows that the energy consumption of opportunistic communication can be further reduced by the cascaded integration of pairwise wakeup periods. When $p \leq 0.4$, most of pairwise wakeup periods are short and non-overlapping, and the difference of energy consumption is very small. However, when $p$ is increased to 0.7 , the energy consumption can be reduced by over $40 \%$, because the length of a pairwise wakeup period can be effectively reduced due to the existence of other wakeup periods having been scheduled earlier. The effects of such reduction diminishes again when $p$ continues to increase and nodes stay awake during most of time.

Figure 13(b) shows that such cascaded integration also impacts the performance of opportunistic communication. Especially when $p \geq 0.8$, the data delivery ratio could be reduced by up to $10 \%$. This performance degradation is mainly caused by our techniques proposed in Section V-B for preserving the scheduling consistency. We used the relaxed probability specified in Eq. (13) for wakeup scheduling, and hence increase the inaccuracy of contact prediction. To summarize, our schemes further reduce the energy consumption if the performance requirement specified by the parameter $p$ is suitably determined, but it could also unnecessarily impair the communication performance with an inappropriate value of $p$. Therefore, the value of $p$ must be carefully chosen according to the practical network scenarios. 


\section{DISCUSSIONS}

\section{A. Clock Synchronization}

Our scheme does not require global or local clock synchronization among mobile nodes. Due to the symmetric nature of node contacts, two nodes $A$ and $B$ always contact each other at the same time. When they schedule their next pairwise wakeup period with each other as described in Section IV, although the values of $t_{L}$ and $t_{0}$ at nodes $A$ and $B$ may be different due to their asynchronous local clocks, the values of $t_{0}-t_{L}$ at $A$ and $B$ will be the same. This ensures that the next pairwise wakeup periods between $A$ and $B$ are identical at both $A$ and $B$, due to the memoryless characteristic of Poisson process.

\section{B. Temporal Heterogeneity}

In practice, contact patterns of mobile nodes may exhibit temporal heterogeneity as suggested in [10], such that the pairwise contact rates of a node may be various during different time periods in a day. For example, a node may contact its contacted neighbors more frequently during daytime than nighttime. In this case, the accuracy of contact prediction in our scheme will decrease when the pairwise contact rate changes. Nevertheless, our proposed technique in Section IV-B is able to adaptively adjust the wakeup scheduling policies during the transitions of node contact patterns.

\section{Overhead Analysis}

The computational overhead of our scheme is mainly produced by arithmetic operations in Section IV, most of which are computationally inexpensive. These computations are only needed every time nodes contact each other. Since inter-contact time is much longer than contact durations, such computational overhead is negligible during the network lifespan.

The communication and storage overhead of our scheme is also affordable. A node only needs to maintain the pairwise contact rates with its contacted neighbors, as well as its current cumulative wakeup schedule. A node only needs to transmit such information to its contacted neighbors every time when it schedules the next pairwise wakeup period, to preserve scheduling consistency as described in Section V-B. This communication overhead is hence considered as negligible.

\section{CONCLUSION}

In this paper, we propose techniques to adaptively schedule wakeup periods of mobile nodes between their inter-contact times, which are much longer than their contact durations. A node stays asleep during inter-contact times when contact probing is unnecessary, and hence significantly reduces the energy consumption of contact probing. We probabilistically predict node contacts in the future, and analytically balance between energy consumption and performance of opportunistic communication. Extensive simulations show that our approach significantly improves the energy efficiency of opportunistic communication. Future work includes more detailed evaluation of contact prediction error, as well as the evaluation of energy saving effectiveness in dense mobile networks where intercontact time is much shorter. We will also investigate more realistic network scenarios where mobile nodes exhibit more dynamic and heterogeneous behaviors and contact patterns.

\section{REFERENCES}

[1] A. Balasubramanian, B. Levine, and A. Venkataramani. DTN Routing As a Resource Allocation Problem. In Proc. SIGCOMM, 2007.

[2] N. Banerjee, M. Corner, and B. Levine. An energy-efficient architecture for dtn throwboxes. In Proceedings of INFOCOM, pages 776-784, 2007.

[3] H. Cai and D. Y. Eun. Crossing over the bounded domain: from exponential to power-law inter-meeting time in manet. Proc. MobiCom, pages 159-170, 2007.

[4] B. J. Choi and X. Shen. Adaptive asynchronous sleep scheduling protocols for delay tolerant networks. IEEE Transactions on Mobile Computing, 10(9):1283-1296, 2011.

[5] H. Dubois-Ferriere, M. Grossglauser, and M. Vetterli. Age matters: efficient route discovery in mobile ad hoc networks using encounter ages. Proc. MobiHoc, pages 257-266, 2003.

[6] N. Eagle and A. Pentland. Reality mining: sensing complex social systems. Personal and Ubiquitous Computing, 10(4):255-268, 2006.

[7] J. Eriksson, L. Girod, B. Hull, R. Newton, S. Madden, and H. Balakrishnan. The Pothole Patrol: Using a Mobile Sensor Network for Road Surface Monitoring. In Proceeding of MobiSys. ACM, 2008.

[8] V. Erramilli, M. Crovella, A. Chaintreau, and C. Diot. Delegation forwarding. In Proceedings of MobiHoc, pages 251-260, 2008.

[9] K. Fall. A Delay-Tolerant Network Architecture for Challenged Internets. Proc. SIGCOMM, pages 27-34, 2003.

[10] W. Gao and G. Cao. On Exploiting Transient Contact Patterns for Data Forwarding in Delay Tolerant Networks. In Proceedings of ICNP, pages 193-202, 2010

[11] W. Gao, G. Cao, A. Iyengar, and M. Srivatsa. Supporting cooperative caching in disruption tolerant networks. In Proceedings of Int'l Conf. on Distributed Computing Systems (ICDCS), 2011.

[12] W. Gao, Q. Li, B. Zhao, and G. Cao. Multicasting in delay tolerant networks: a social network perspective. In Proceedings of MobiHoc, pages 299-308, 2009.

[13] S. Guo, Y. Gu, B. Jiang, and T. He. Opportunistic flooding in low-dutycycle wireless sensor networks with unreliable links. In Proceedings of MobiCom, pages 133-144, 2009.

[14] P. Hui, A. Chaintreau, J. Scott, R. Gass, J. Crowcroft, and C. Diot. Pocket switched networks and human mobility in conference environments. In Proceedings of the 2005 ACM SIGCOMM workshop on DelayTolerant Networking (WDTN), pages 244-251. ACM, 2005.

[15] P. Hui, J. Crowcroft, and E. Yoneki. Bubble rap: social-based forwarding in delay tolerant networks. Proc. MobiHoc, 2008.

[16] H. Jun, M. H. Ammar, M. D. Corner, and E. W. Zegura. Hierarchical power management in disruption tolerant networks with traffic-aware optimization. In Proceedings of the 2006 SIGCOMM Workshop on Challenged Networks (CHANTS), pages 245-252. ACM, 2006.

[17] F. Li and J. Wu. Mops: Providing content-based service in disruptiontolerant networks. In Proceedings of Int'l Conf. on Distributed Computing Systems (ICDCS), pages 526-533, 2009.

[18] Y. Li, Y. Jiang, D. Jin, L. Su, L. Zeng, and D. Wu. Energy-efficient optimal opportunistic forwarding for delay-tolerant networks. IEEE Transactions on Vehicular Technology, 59(9):4500-4512, 2010.

[19] L. McNamara, C. Mascolo, and L. Capra. Media sharing based on colocation prediction in urban transport. In Proceedings of MobiCom, pages 58-69, 2008.

[20] M. McNett and G. Voelker. Access and mobility of wireless PDA users. ACM SIGMOBILE CCR, 9(2):40-55, 2005.

[21] T. Spyropoulos, K. Psounis, and C. Raghavendra. Spray-and-focus: Efficient mobility-assisted routing for heterogeneous and correlated mobility. In Pervasive Computing and Communications Workshops, pages 79-85, 2007.

[22] W. Wang, V. Srinivasan, and M. Motani. Adaptive contact probing mechanisms for delay tolerant applications. In Proceedings of MobiCom, pages 230-241. ACM, 2007.

[23] Y. Xi, M. Chuah, and K. Chang. Performance evaluation of a power management scheme for disruption tolerant networks. Mobile Networks and Applications, 12(5):370-380, 2007.

[24] Q. Yuan, I. Cardei, and J. Wu. Predict and relay: an efficient routing in disruption-tolerant networks. In Proceedings of MobiHoc.

[25] H. Zhu, L. Fu, G. Xue, Y. Zhu, M. Li, and L. M. Ni. Recognizing Exponential Inter-Contact Time in VANETs. In Proc. INFOCOM, 2010. 